\title{
Strategic encoding and enhanced memory for positive value-location associations
}

\author{
Shawn T. Schwartz ${ }^{1}$ (D) Alexander L. M. Siegel ${ }^{1}$ (D) Alan D. Castel $^{1}$ (D) \\ Published online: 8 April 2020 \\ (C) The Psychonomic Society, Inc. 2020
}

\begin{abstract}
People often need to remember the location of important objects or events, and also to remember locations that are associated with negative objects. In the current study, we examined how both positive and negative items might be selectively remembered in the visuospatial domain. Participants studied number-items ranging from -25 to +25 indicating point values in a grid display and were instructed to maximize their score (a summation of correctly remembered positive and negative information; incorrectly placed negative items resulted in a subtraction from the overall score). Items were presented in a sequential, simultaneous (Experiment 1), or self-regulated format (Experiment 2) where participants controlled which items to study and the length of study time per item. In Experiment 1, participants selectively recalled high-magnitude over low-magnitude items, but also displayed a positivity preference in memory. In Experiment 2, we were able to determine whether this positivity preference was a result of bottom-up, automatic, or top-down strategic processes. Results indicated that participants explicitly chose to study positive items more frequently and for more total time relative to negative items, suggesting a deliberate strategy to focus on positive information. This bias for highly positive information suggests an overt points-gained approach, as opposed to a lossaversion approach, to remembering value in the visuospatial domain.
\end{abstract}

Keywords Associative memory $\cdot$ Positivity $\cdot$ Negativity $\cdot$ Presentation format $\cdot$ Visuospatial memory

\section{Introduction}

The ability to remember the locations of items is one form of visuospatial memory. Much like other forms of memory, capacity constraints limit the amount of visuospatial information that we remember, with visuospatial memory capacity similar to that of verbal memory (Kane et al., 2004; Park et al., 2002). Furthermore, visuospatial memory often involves the binding of identity (visual) to location (spatial) information (Chalfonte \& Johnson, 1996; Thomas, Bonura, Taylor, \& Brunyé, 2012). That is, it is usually important to remember not only the visual features of the item (e.g., the front of the restaurant, the signage, etc.) or the location features (e.g., where it is located in

Electronic supplementary material The online version of this article (https://doi.org/10.3758/s13421-020-01034-4) contains supplementary material, which is available to authorized users.

Shawn T. Schwartz

shawnschwartz@ucla.edu

11285 Psychology Tower, Department of Psychology, University of California, Los Angeles, Los Angeles, CA 90095, USA town, what stores are adjacent, etc.), but also the linkage between these features (e.g., that this particular restaurant is in this particular location). The need to bind multiple features into a coherent unit in memory represents a form of associative memory that may be fairly cognitively demanding relative to memory for single features (Shing et al., 2010) and that typically suffers marked deficits in cognitive healthy aging (associative deficit hypothesis; Naveh-Benjamin, 2000; Old \& Naveh-Benjamin, 2008) and pathological aging, as in Alzheimer's disease (Gallo, Sullivan, Daffner, Schacter, \& Budson, 2004).

Despite the demanding nature of associative binding, similar to memory for individual units of information, people can selectively attend to and prioritize associations in memory based on their value. This finding, referred to as valuedirected remembering (VDR; Castel, Benjamin, Craik, \& Watkins, 2002; Nguyen, Marini, Zacharczuk, Llano, \& Mudar, 2019; Stefanidi, Ellis, \& Brewer, 2018), has been found in both the verbal memory domain using unrelated word pairs as stimuli (Ariel, Price, \& Hertzog, 2015) and the visuospatial memory domain using item-location associations (Siegel \& Castel, 2018a, 2018b). The ability to selectively prioritize information in memory is maintained under various 
conditions in which cognitive resources are depleted, which includes dual-task paradigms where a secondary distractor task is present during encoding (Middlebrooks \& Castel, 2018; Siegel \& Castel, 2018b) and to a certain extent in children with attention-deficit hyperactivity disorder (ADHD; Castel, Lee, Humphreys, \& Moore, 2011) and older adults with Alzheimer's disease (Castel, Balota, \& McCabe, 2009; Wong et al., 2019; cf. Elliott \& Brewer, 2019, who found that selectivity was impaired under dual-task conditions in a recognition memory task). These findings indicate that when capacity - either working-memory capacity as in dual-task paradigms or short-term memory capacity as in aging and cognitive impairment - is exceeded, the prioritization of information remains fairly robust and relatively insensitive to changes in the amount of available cognitive resources.

Importantly, prior work investigating this prioritization of information based on value characteristics has primarily examined this effect under conditions when points can be gained, not lost. That is, in a typical associative VDR task, participants are instructed that a particular word pair or itemlocation pair is worth a certain amount of points if correctly remembered or correctly replaced in a location (e.g., CHAIRDOG -8 points, hammer in top left corner of grid array -3 points, for words and items that range in point values from 1 to 10). Participants are instructed to maximize their point score, which leads to an attentional focus on and better memory for high-value over low-value information (Castel et al., 2002; Middlebrooks, Kerr, \& Castel, 2017; Siegel \& Castel, 2018a). In these studies, and others like them, however, participants are not penalized if the paired associate is not recalled or an item is misplaced during the testing phase. As such, participants can truly focus on the prioritization of the highest value information, without much consideration for items of lesser value. In real-world situations, information we seek to remember can be highly positive, highly negative, or somewhere in between. Other empirical work has suggested that subjective valuations associated with losses may be different to those associated with gains (Mitchell \& Wilson, 2010), which may extend to participants' strategy use and subsequent memory performance. As such, the current study sought to systematically examine how participants prioritize information when misremembering is penalized, by introducing the potential to lose points.

Some work has investigated how participants study information of differing value with the potential of point loss. For example, in an application of associative VDR using more naturalistic materials, Castel et al. (2016) presented participants with faces randomly paired with monetary values ranging in magnitude from $-\$ 100$ to $+\$ 100$ (values presented included 1, 2, 5, 10, 20, 50, and 100). Positive values indicated fictional money that was owed to them, while negative values indicated money that they owed. This study found that the extreme values (i.e., those higher in overall magnitude) were better recalled than those of smaller magnitude, regardless of positivity and negativity. Other related work also suggests that extreme values rather than exclusively high positive rewards may enhance memory performance due to their bottom-up salience (Madan, Ludvig, \& Spetch, 2014; Madan \& Spetch, 2012). This has been supported by various neuroimaging studies showing reward-processing brain regions - such as the nucleus accumbens and striatum - automatically respond to reward saliency (Cohen, Rissman, Suthana, Castel, \& Knowlton, 2014; Cooper \& Knutson, 2008; Shigemune, Tsukiura, Kambara, \& Kawashima, 2013; Zink, Pagnoni, Martin-Skurski, Chappelow, \& Berns, 2004).

While the aforementioned work was primarily interested in the automatic, bottom-up effects of reward on memory performance, the current investigation sought to explore how participants attempt to study positive and negative information in a more top-down, controlled manner. Prior studies have investigated how participants allocate attention to information differing in positive value, with only the potential to gain points (e.g., Middlebrooks \& Castel, 2018). Perhaps unsurprisingly, when participants were afforded more control over their study choices and study time, they were more selective in their memory for high-value items, indicating more efficient prioritization relative to when they were given less control. This finding suggests that when top-down strategic processing can be more effectively implemented, participants choose to focus on high-value information. However, it is currently unclear how the presence of high-value negative information may or may not influence participants' study choices and value-based memory.

The potential influence of losing points on one's valuebased study strategies is a theoretically interesting avenue to explore. As proposed by the seminal prospect theory (Kahneman \& Tversky, 1979; Tversky \& Kahneman, 1992), people tend to be risk averse when making financial decisions about potential gains (e.g., they would rather have a 100\% chance at winning $\$ 50$ than a $50 \%$ chance at winning $\$ 100$ ), while they are risk seeking in terms of potential losses (e.g., they would rather choose a $50 \%$ chance at losing $\$ 100$ or losing $\$ 50$ than a $100 \%$ chance at losing $\$ 50$ ). With this theory, they were able to account for the finding that "losses loom larger than gains" - that is, the subjective feeling of losing $\$ 50$ is more highly negative than the subjective feeling of winning $\$ 50$ is positive. As such, prospect theory predicts that participants should adopt a point-loss avoidance approach in the current study, as negative point values may receive more attentional focus and better subsequent memory.

Another theoretical framework that provides different predictions regarding participants' value-based study strategies in the presence of positive and negative information is the regulatory fit theory (Higgins, 2005, 2006; Spiegel, Grant-Pillow, \& Higgins, 2004). Regulatory fit theory proposes that participants' goal orientation, which is dependent on individual and 
situational factors, leads to different types of goal pursuit, such as a promotion-focused or prevention-focused goal orientation (Cesario, Higgins, \& Scholer, 2007; Higgins, 2006). Therefore, engagement in and success on a task may be driven by the goal orientation that participants are directed to follow. In the current study, given that participants are prescribed a particular motivation orientation to pursue at the beginning of the task (i.e., to maximize their score, clearly a promotionfocused orientation), regulatory fit theory predicts a focus on engagement in strategies that pursue this prescribed goal, with participants potentially focusing more on remembering positive items to gain points rather than negative items to avoid losing points. The current study was interested in determining whether regulatory fit would account for participants' adoption of a points-gained approach in accordance with the prescribed goal to maximize the points earned throughout the task. The main goal of the following experiments, then, was to provide evidence of a gain-oriented (as predicted by regulatory fit theory) or loss-avoidance-oriented (as predicted by prospect theory) approach to studying associative information of both positive and negative value.

Further, the effectiveness of participants' strategy implementation may depend on the level of available cognitive resources. Numerous studies have indicated that participants remember more information and are more effective in implementing strategies when information is presented simultaneously (i.e., all at the same time) relative to sequentially (i.e., one at a time; Ariel, Dunlosky, \& Bailey, 2009; Dunlosky \& Thiede, 2004; Middlebrooks \& Castel, 2018; Robison \& Unsworth, 2017; Siegel \& Castel, 2018b). The mechanisms underlying this benefit of simultaneous over sequential presentation include various factors related to the amount of attentional control during encoding. When information is presented sequentially, there is a higher demand on cognitive resources during encoding, as participants have to store previously presented information in working memory and make decisions about whether to allocate attention to upcoming items. During this presentation format, study time for each item is experimenter-allocated, so it is more difficult for participants to make comparisons across items in order to select specific ones to study. Instead, participants are forced to make item-by-item selection decisions, which may distract them from their overall agenda. However, when information is presented simultaneously, participants can more effectively compare across all information, select items that fit their strategy, and return to restudy these items. During this presentation format, since study time for each item is participant-allocated, participants have greater attentional control during encoding.

In the present study, therefore, participants were tested on their ability to remember visuospatial information containing items of both positive $(+25,+20,+15,+10,+5)$ and negative ($25,-20,-15,-10,-5)$ values. As participants encoded and recalled the specific value-location associations, they had the choice to utilize their own strategy (i.e., avoid losing points by correctly recalling negative value locations and/or gaining points by correctly recalling positive value locations), all while attempting to maximize their score. In Experiment 1, selectivity strategies regarding the encoding and retrieval of both positive- and negative-value locations were investigated through varying presentation format. By utilizing multiple formats in Experiment 1, we were able to determine how participants' approaches may be influenced under varying levels of cognitive load, with the assumption that participants would more effectively implement their strategy under simultaneous, relative to sequential, presentation-format conditions due to the increased amount of cognitive control. It is important to note that although bottom-up processes may have a greater influence on memory performance in the sequential format and top-down processes may have a greater influence in the simultaneous format, both types of processing are undoubtedly present in both formats. That is, while memory performance may be driven primarily by item characteristics when sequentially presented, participants are also likely to engage in strategies to remember items (i.e., opting to study some items and not others, engaging in relational/elaborative processing, etc.). On the other hand, simultaneously presented items may allow for more strategic processing, but individual items may also capture attention and influence later memory. As such, the presentation format may not implicate the exclusive influence of either bottom-up or top-down processes on memory, but rather "tips the scale" towards one type of processing. In Experiment 2, participants were given the most control over their study decisions by utilizing a self-selected and self-paced VDR paradigm in which participants chose which items they wanted to study and for how long. This experiment allowed us to more directly examine value-based study strategizing and whether participants were more likely to adopt a gain or loss-avoidance approach.

\section{Experiment 1}

In Experiment 1, participants studied sequentially or simultaneously presented items represented by positive and negative numerical values in a $5 \times 5$ grid. We were interested in how participants would approach studying these items of varying magnitude and how this might change with both increasing task experience (across multiple trials) and varying levels of attentional resources (by manipulating presentation format). After viewing the study grid, participants were given a memory test in which they were required to place items into their previously viewed locations and were immediately given detailed feedback on their performance, including which positive and negative items were correctly or incorrectly placed and their total score. Participants then repeated this procedure for a total of eight study-test cycles, with both positive and 
negative value-location pairs appearing in each grid. Prior research has revealed the importance of examining across multiple trials for assessing strategy optimization coupled with increasing task experience (Ariel \& Dunlosky, 2013; Castel, 2008; Middlebrooks et al., 2017; Nelson \& Narens, 1990; Siegel \& Castel, 2018b; Wong et al., 2019). This motivated the utilization of eight study-test cycles in the current study as detailed feedback was provided at the end of each study-test cycle so that participants could incorporate it and modify their study strategies on this value-based, goaldirected task. We hypothesized, then, that with increased task experience and feedback, participants may engage in more effective encoding strategies and earn more points on each successive trial.

With regard to memory for positive and negative items, we hypothesized that participants would remember higher overall magnitudes (regardless of sign) better than lower magnitudes. Further, we expected that participants would remember positive and negative information equally, consistent with prior work demonstrating that both rewards and punishments may produce equivalent memory enhancement (Castel et al., 2016; Madan et al., 2014; Shigemune et al., 2013). However, this proposed equivalence between negative and positive items may only be the case when participants are able to effectively engage in strategic control processes during encoding (as in the simultaneous presentation format) with a more efficient, top-down-directed implementation of strategy. Yet, when resources are more strained during encoding (as in the sequential format), top-down strategic processes may be less effective, and the bottom-up influence of particular item characteristics may have a greater effect on memory performance. One potential hypothesis is that participants' attention may be more captured by negative items, as the losses may loom larger than the gains, in line with prospect theory (Kahneman \& Tversky, 1979; Tversky \& Kahneman, 1992). On the other hand, as predicted by the regulatory fit theory (Higgins, 2005, 2006; Spiegel et al., 2004), participants in this demanding sequential format may adopt a points-gained approach consistent with the phrasing of task goals to maximize points earned, focusing on and remembering more positive item-locations.

\section{Method}

Participants One-hundred and ten younger adults ( 84 females, $M_{\text {age }}=20.78$ years, $S D_{\text {age }}=1.50$ years, age range: $18-27$ years), randomly assigned into two experimental presentation conditions: sequential ( $n=55$, 45 females, $M_{\text {age }}=20.65$ years, $S D_{\text {age }}=1.68$ years, age range: $18-27$ years) and simultaneous ( $n=55,39$ females, $M_{\text {age }}=20.91$ years, $S D_{\text {age }}=1.29$ years, age range: $18-24$ years), volunteered to participate in this study. All participants were University of California, Los Angeles (UCLA) undergraduate students who participated for course credit. All participants presented with normal or corrected-to-normal vision, no physical disability, and clinically normal cognitive function.

Materials and apparatus The items used as stimuli in this study were designed in Adobe Photoshop and were ten simple numerical values ranging from -25 to +25 , in increments of 5 with no 0 value (Fig. 1). On the computer screen, each item was $200 \times 200$ px in size, consisting of text typed in "Open Sans" bold-weight font, size 106.1 pt, colors: \#b80001 (red, negative items) and \#02a747 (green, positive items). Each of the presentation orders (sequential) and assigned locations (sequential, simultaneous) of the number-items was pseudorandomized and placed within a $5 \times 5$ grid with the constraint that no more than two items be present in any row or column (to avoid arbitrarily forming spatial patterns that may aid memory). On the computer screen, the size of each grid was $550 \times 550 \mathrm{px}$ (with each cell $110 \times 110 \mathrm{px}$ in size). Each item and its number displayed represented the locations' value ranging from -25 (lowest value) to +25 (highest value). This process of adding the number-items to different spatial locations within each study grid was repeated to form eight unique grids each with a different arrangement of the ten number-items (e.g., the -20 item was in a different location on each of the eight trials). In order to prevent against testing effects, the locations of the positive and negative item values within each grid were completely randomized per trial and per

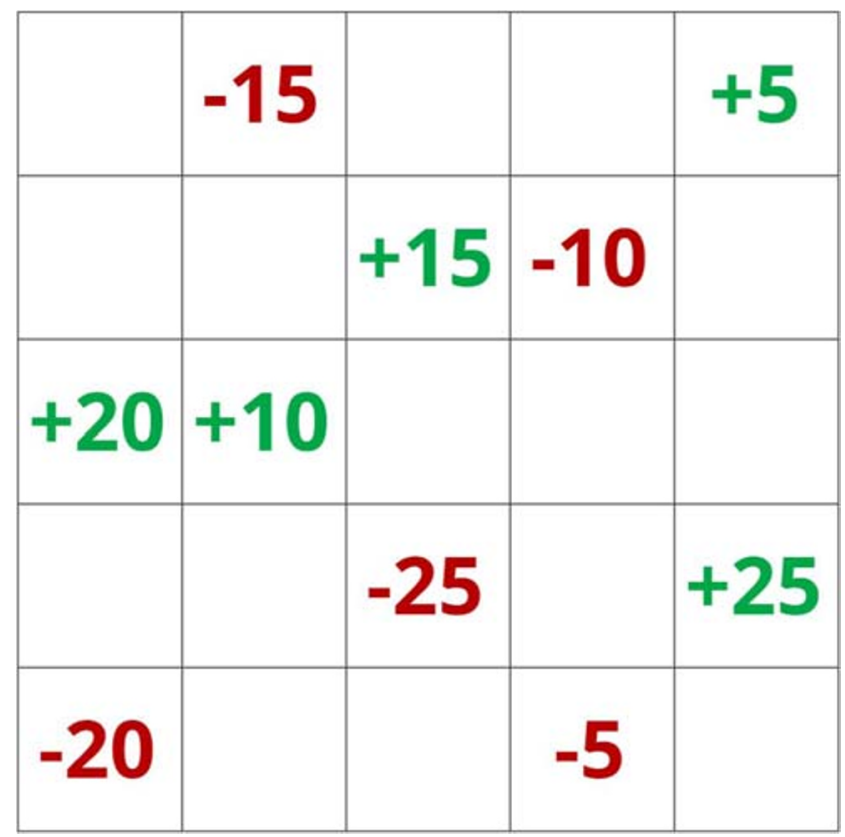

Fig. 1 An example of a grid that participants may have been presented with during the simultaneous study phase (Experiment 1 ). Ten values (five positive, five negative), ranging from -25 to +25 in increments of 5 , excluding 0 , were randomly assigned a location in the grid. No row or column had more than two values. In Experiment 1, values were presented sequentially or simultaneously, as seen in this figure 
participant. That is, while one participant may have been presented with a +25 number-item in the top left cell of the third grid, that same +25 number-item could have been located in the bottom right cell of the seventh grid for a different participant. Positive value items were always green and negative value items were always red. As such, each participant was presented with a different set of eight completely randomized study grids.

Procedure The procedure used in this study was based upon methodologies used in prior experiments investigating VDR (e.g., Castel et al., 2002; Hayes, Kelly, \& Smith, 2013; Robison \& Unsworth, 2017) and visuospatial memory (e.g., Chalfonte \& Johnson, 1996; Thomas et al., 2012; Siegel \& Castel, 2018a, 2018b). Participants were instructed that they would be shown a grid with various numbers placed throughout the grid's cells and to remember the locations of the values for a later test. They were then instructed that the numbers presented within the grid would differ in value, ranging from -25 (lowest value) to +25 (highest value), in increments of 5 , excluding 0 , indicated by the number of the item in the cell, and that their goal would be to maximize their score (a summation of the points associated with a correctly remembered item). Participants were also instructed that the penalty for misplacing negatively valued number-items would be losing that value from their overall score. For example, if the participant correctly placed a number that was worth +25 points, they would receive +25 points towards their total score. If they correctly placed a number that was worth -10 points, they would avoid losing 10 points from their total score. For the sequential presentation format, participants were shown each number-item for $3 \mathrm{~s}$ (totaling $30 \mathrm{~s}$ for the ten presented number-items). There was no inter-stimulus interval in between each stimulus presentation as sequentially presented items were shown with each preceding item disappearing directly before the appearance of the next item. For the simultaneous presentation format, participants were instructed that they would see all ten number-items within the grid at the same time, while studying the grid for a total of $30 \mathrm{~s}$.

After viewing the grid, participants were shown a brief visual mask for $0.5 \mathrm{~s}$ and then a blank $5 \times 5$ grid with the previously presented number-items in a row underneath. Participants were instructed to replace the items in their previously viewed locations using the computer mouse. If unsure about a value's location, participants were instructed to make a guess at its location. There was no time limit for participants during the testing phase. After participants placed all ten items, they were given immediate feedback both on their total score (out of 75 points per grid) and the number of points gained (by correctly placing positively valued number-items), lost (by incorrectly placing negatively valued number-items), failed-to-gain (by incorrectly placing positively valued number-items), and avoided-losing (by correctly placing negatively valued number-items). Participants were able to review their feedback for as long as they desired and were instructed to click a button to advance them to the next grid when they felt ready to do so. After choosing to advance, the subsequent trial would commence with participants immediately shown the new grid to study. Participants then repeated this procedure for all eight grids. After conclusion of the eight study-test cycles, the experiment was completed.

All materials and procedures used in the current study were approved by the UCLA Institutional Review Board.

\section{Results}

Scoring performance Participants had the opportunity to score a minimum of -75 points and a maximum of +75 points in each study-test cycle. To examine their overall scoring performance with regard to grid number between the two presentation formats, we conducted a 2 (Presentation format: sequential, simultaneous) $\times 8$ (Grid number: $1,2, \ldots, 8)$ mixed analysis of variance (ANOVA) on the overall points-scores. Grid number was included as a factor in this and later analyses as prior research has consistently demonstrated that participants may not optimally execute a value-based study strategy on the first trial, but increase their performance with continued task experience and feedback (Castel, 2008; Middlebrooks et al., 2017; Siegel \& Castel, 2018a).

This analysis revealed a significant main effect of presentation format, $F(1,108)=47.49, p<.001, \eta^{2}=.31$, with participants in the simultaneous condition scoring relatively higher $(M=-0.62, S D=1.91)$ than participants in the sequential condition $(M=-2.90, S D=1.55), t(108)=6.89$. A significant main effect of grid number was revealed, $F(7,756)=$ 2.46, $p=.02, \eta^{2}=.02$, but follow-up post hoc independent samples $t$-tests with a Bonferroni correction revealed no significant differences (all adjusted $p s>.24$ ). Finally, there was no interaction between presentation format and grid number, $F(7,756)=0.34, p=.94, \eta^{2}=.003$.

To further examine the potential presence of a linear or quadratic trend between grid number and points-scores despite no significant differences in Bonferroni-corrected $t$-tests, we conducted a polynomial regression predicting pointsscores from grid number averaged between presentation formats (given the lack of format $\times$ grid number interaction). The regression model took the following form: Points $=\beta_{0}+\beta_{1}$ (Grid number $)+\beta_{2}$ (Grid number $)^{2}$. The continuous predictor grid number was entered into the model as a mean-centered variable. The quadratic term was entered into the model to account for the possibility of a U-shaped relationship between grid number and points score (i.e., potentially higher performance at the beginning and end of the task). The model was a significant predictor of points-scores, $R^{2}=.01, F(2,877)=$ 5.07, $p=.01$. Both the coefficients for the intercept, $\beta_{0}=$ $1.73, p<.001$, and the linear term, $\beta_{1}=.17, p=.002$, were 
significant predictors, while the quadratic term coefficient was not, $\beta_{2}=-.01, p=.83$. This finding indicates a positive linear relationship between grid number and points such that with each increase in grid number the amount of points earned also increased.

Memory performance Overall memory performance was assessed by the ability of participants to correctly replace values into the exact target locations in which they were viewed in the prior study phase for each grid. Error magnitude (i.e., how many cells away an item was misplaced) was also examined as a function of grids and item value. These results were largely consistent with those examining correct recall performance described below and are included in the Online Supplementary Materials.

Recall across grids To examine overall memory performance with increasing task-experience when number-items were presented either sequentially or simultaneously and regardless of item value, we conducted a 2 (Presentation format: sequential, simultaneous) $\times 8$ (Grid number: $1,2, \ldots, 8)$ mixed ANOVA on the proportion of items correctly placed (out of 10 possible items; Fig. 2). This analysis revealed a significant main effect of presentation format, $F(1,108)=55.29, p<.001, \eta^{2}=.34$, such that participants in the simultaneous condition $(M=.45$, $S D=.12$ ) correctly replaced a greater proportion of items, as compared to participants in the sequential condition $(M=.29$, $S D=.09)$. There was no main effect of grid number, $F(7,756)$ $=1.88, p=.07, \eta^{2}=.02$, and no significant interaction between presentation format and grid number, $F(7,756)=0.31$, $p=.95, \eta^{2}=.003$.

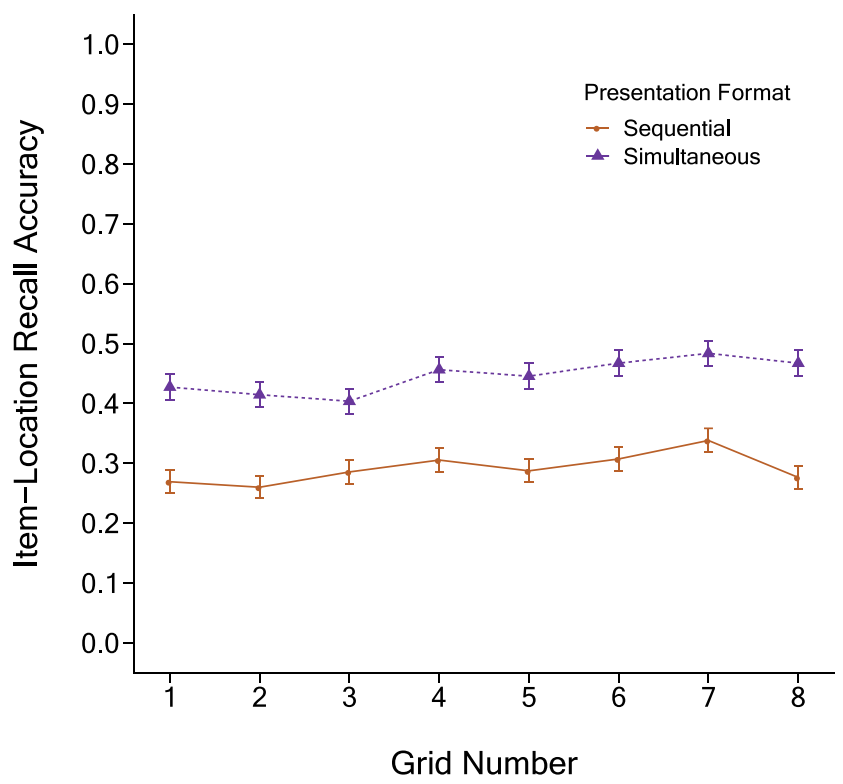

Fig. 2 The proportion of number-items correctly placed as a function of grid number when presented sequentially or simultaneously in Experiment 1 . Error bars represent \pm 1 standard error
Recall by item value To examine overall memory performance as a function of item values between presentation formats, we conducted a 2 (Presentation format: sequential, simultaneous) $\times 10$ (Item value: $-25,-20, \ldots,+25$ ) mixed ANOVA on the proportion of items correctly placed (Fig. 3). Mauchly's Test of Sphericity indicated that the assumption of sphericity had been violated, $\chi^{2}(44)=141.36, p<.001$; therefore, a Greenhouse-Geisser $(\varepsilon=.76)$ correction was used. The previously described main effect of presentation format was found again, $F(1,108)=55.29, p<.001, \eta^{2}=.34$. There was also a significant main effect of item value, $F(6.81,735.33)=12.80$, $p<.001, \eta^{2}=.11$, but no interaction between item value and presentation format, $F(6.81,735.33)=1.09, p=.37, \eta^{2}=.01$.

The significant main effect of item value suggests that item value differentially influenced recall accuracy. Assessing the relationship between recall accuracy and item value in an ANOVA framework would require many post hoc comparisons due to the number of item value pairs, thus reducing our ability to detect any significant differences. Instead, we conducted linear and quadratic model fits for memory performance as a function of item value in a regression framework to examine overall trends. As the previously described ANOVA indicated no significant difference between presentation formats in terms of the relationship between item value and recall, we collapsed across these conditions in the following regressions. Tested linear models were of the following form: Recall Accuracy $=\beta_{0}+\beta_{1}$ (item value). Tested quadratic models were of the following form: Recall Accuracy = $\beta_{0}+\beta_{1}$ (item value) $+\beta_{2}$ (item value) ${ }^{2}$.

The quadratic model was significant, $R^{2}=.81, F(2,9)=$ $15.35, p=.003$, with the following standardized coefficients,

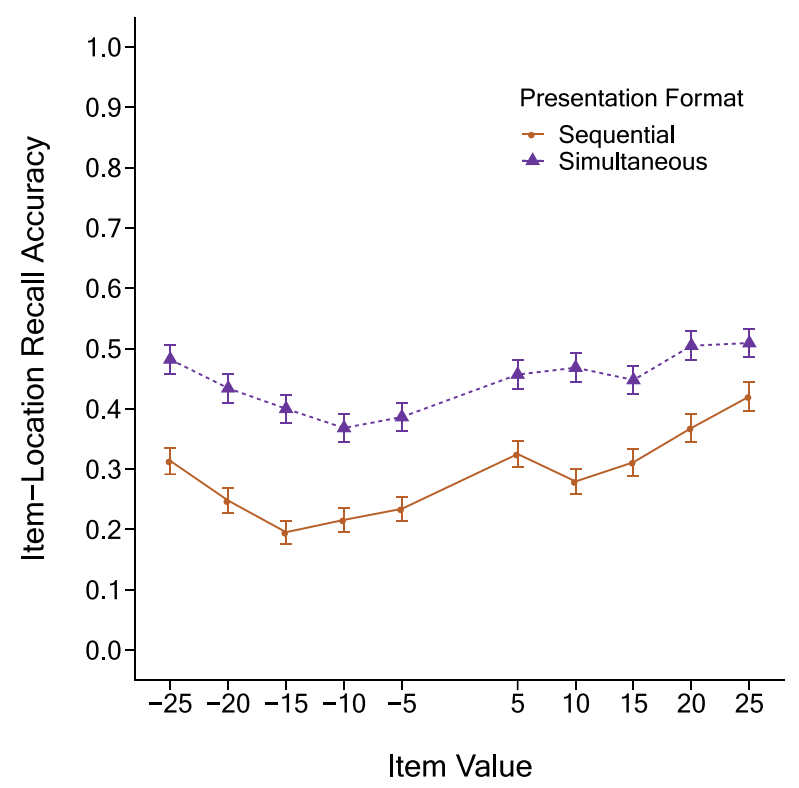

Fig. 3 The proportion of number-items correctly placed as a function of item value when presented sequentially or simultaneously in Experiment 1. Error bars represent \pm 1 standard error 
$\beta_{1}=.66, p=.01$, and $\beta_{2}=.62, p=.01$, indicating a U-shaped relationship between item value and recall accuracy. This result indicates that more extreme values (i.e., those closer to \pm 25 ) were better remembered than more median values (those closer to \pm 5 ) in both presentation formats.

Recall by sign In addition to looking at the overall trends through regression analyses, we were also interested in examining the differences between positive and negative values of the same magnitude for each of our dependent measures (e.g., comparing recall between -25 and +25 ). While our regression analyses allowed us to determine overall trends, they did not reveal the individual differences between the positive and negative values of the same magnitude. Thus, to determine whether there was a bias for positive or negative values, we conducted paired-samples $t$-tests with a Bonferroni correction collapsed across grids. For each item magnitude, the positive value (e.g., +20) was recalled more accurately than the corresponding negative value (e.g., -20$)$, adjusted $p \mathrm{~s}<.03$. Overall, there was higher recall accuracy for positive $(M=.41, S D=$ $.15)$ relative to negative $(M=.33, S D=.15)$ items, $t(109)=$ $5.65, p<.001$.

Response order by item value Another way of examining participants' strategies was to examine the order in which they output items. If a participant's strategy was to remember negative or positive items first, then this would likely be reflected in their recall order, with those items placed earlier in the recall phase (Middlebrooks \& Castel, 2018). In the context of the current task, as participants were required to place all ten items before proceeding to the next trial, we were able to analyze the order in which information was outputted and whether this varied as a function of presentation format.

To examine the order in which they replaced each item into the test grid, we conducted a 2 (Presentation format: sequential, simultaneous) $\times 10$ (Item value: $-25,-20, \ldots,+25$ ) mixed ANOVA on response (output) order (Fig. 4). Output order ranged from 1 (the first item placed during the recall phase) to 10 (the last item placed during the recall phase), with lower scores indicating an earlier output and higher scores indicating a later output. Given that participants were able to move items around in the grid at their discretion (i.e., an item was not "locked in" after its first placement), we used the final output position for each item. For example, if participants placed all ten items and then shifted the item that they had placed fourth to a new location, that item would then become the last item placed and receive an output order score of 10 . This output order variable was used as the outcome variable in the following analyses. Mauchly's Test of Sphericity indicated that the assumption of sphericity had been violated, $\chi^{2}(44)=342.79, p$ $<.001$; therefore, a Greenhouse-Geisser $(\varepsilon=.505)$ correction was used. There was a significant main effect of item value, $F(4.55,490.93)=19.66, p<.001, \eta^{2}=.15$, and a significant interaction between item value and presentation format, $F(4.55,490.93)=2.91, p=.02, \eta^{2}=.02$.

Given the significant interaction, the relationship between item value and response order was analyzed separately within each presentation format using the same linear and quadratic models described in the recall-by-item-value section. In both presentation formats, a significant quadratic relationship was found indicating an inverted U-shape relationship between item value and response order, $R^{2}=.84, F(2,9)=18.48, p=$ .002 , and $R^{2}=.81, F(2,9)=15.55, p=.003$, for the sequential and simultaneous formats, respectively. In each model, both the linear $\left(\beta_{\text {Seq }}=-.77, p=.001\right.$ and $\left.\beta_{\text {Sim }}=-.73, p=.003\right)$ and the quadratic $\left(\beta_{\text {Seq }}=-.50, p=.01\right.$ and $\left.\beta_{\text {Sim }}=-.53, p=.01\right)$ standardized coefficients were significant. As such, participants in both presentation formats placed items of higher magnitude (regardless of sign) earlier in the testing phase than items of lower magnitude.

To determine if response order varied as a function of sign (i.e., between negative and positive items), for items presented sequentially, response orders for items of all magnitudes were significantly different compared to their respective counterparts (adjusted $p s<.001$ ), with positively valued items placed before negatively valued items. For items presented simultaneously, items of value +15 were placed earlier than those valued $-15(p<.001)$, while the remaining items of magnitudes $5,10,20$, and 25 did not differ in response order between positive and negative values (adjusted $p \mathrm{~s}>.09$ ). Further, a 2 (Presentation format: sequential, simultaneous)

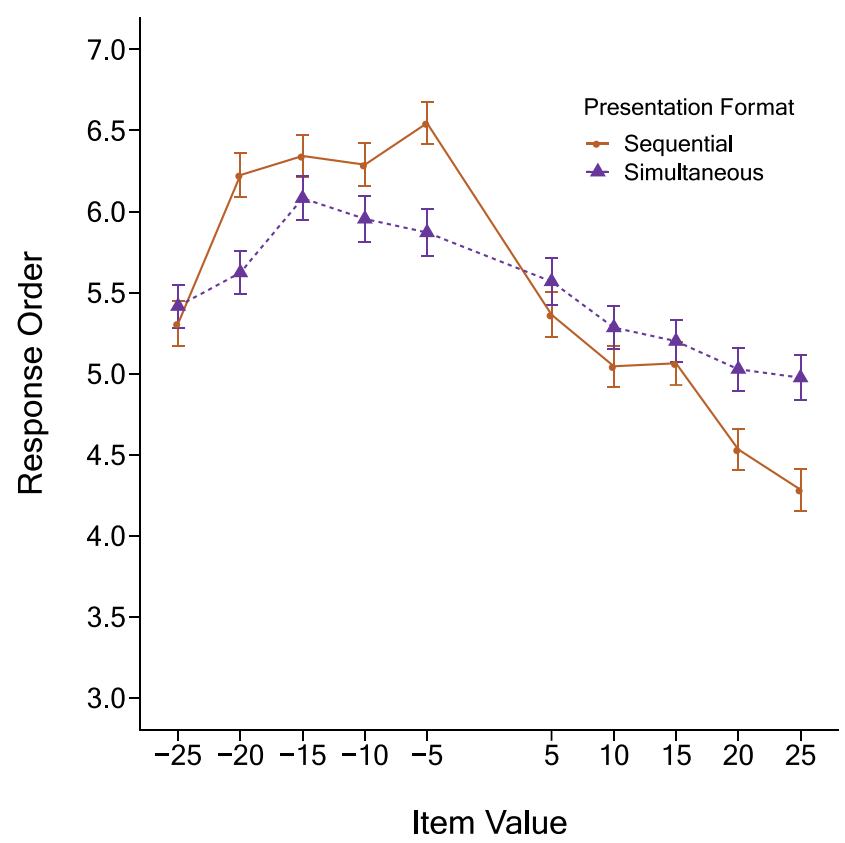

Fig. 4 Mean response order for number-items during the testing phase as a function of item value when presented sequentially or simultaneously in Experiment 1. All error bars represent \pm 1 standard error 
$\times 2$ (Sign: positive, negative) mixed ANOVA on response order revealed a significant interaction, $F(1,108)=5.61, p=$ $.02, \eta^{2}=.04$. Follow-up paired-samples $t$-tests to examine response order differences within each presentation format were conducted. For the sequential format, positive items ( $M$ $=4.86, S D=0.81)$ were replaced earlier in the testing phase than negative items $(M=6.14, S D=0.81), t(54)=5.90, p<$ .001 . This was also the case for the simultaneous format $\left(M_{P o s}\right.$ $\left.=5.21, S D_{P o s}=0.76, M_{N e g}=5.79, S D_{N e g}=0.76\right), t(54)=2.84$, $p=.01$. This indicates that while both presentation formats resulted in earlier placement of positive relative to negative items overall, this difference was larger in the sequential relative to the simultaneous format.

\section{Discussion}

Simultaneously presented information was more accurately recalled compared to information presented sequentially which led to higher point totals in the simultaneous presentation format. While there was better memory for extreme values (e.g., \pm 25 ) in both presentation formats, participants more accurately recalled positive items relative to negative items, suggesting a surprising positivity bias for recall accuracy. Examining response order as an indicator of participants' strategy use indicated that all participants attempted to place positive items before negative items, although this preference appeared to be greater for sequentially presented items. This may have been due to participants attempting to recall as many positive items as they could, thus evidencing a positivity-first, points-gained approach for the more demanding sequential presentation format that reduces top-down influence relative to the simultaneous presentation format. Overall, these results demonstrate preferential treatment of positive items relative to negative items under varying degrees of attentional load during encoding despite their equivalent influence on participants' total score. This evident points-gained approach was further explored in Experiment 2 in which participants had complete control over their study choices.

\section{Experiment 2}

The resulting positivity bias from Experiment 1 prompted Experiment 2 to further investigate the selective control strategies being employed by participants for the prior sequential and simultaneous visuospatial memory tasks. As demonstrated in Experiment 1, participants selectively encoded positive value locations with the highest priority over negative value locations; however, better understanding the strategies that participants utilized to selectively encode these important items required the implementation of a self-regulated study task. Self-regulated paradigms allow participants to more efficiently implement study strategies by giving them the choice to allocate attention and study time to particular items and effectively ignore other items (Castel, Murayama, Friedman, McGillivray, \& Link, 2013; Dunlosky, Ariel, \& Thiede, 2011; Middlebrooks \& Castel, 2018). As such, participants in Experiment 2 not only chose which value-location associations to study during the encoding-phase, but also the duration and frequency of study.

By allowing participants to control their study choices, we could directly examine whether the positivity bias observed in Experiment 1 was more heavily influenced by an overt, topdown strategy enacted by participants or bottom-up, item characteristics. If a pattern similar to Experiment 1 is observed, then it can be inferred that participants actively and selectively attend to and encode positive items over negative items. However, if this difference is eliminated and positive and negative items are remembered at an equivalent rate, then the results of Experiment 1 can be attributed to the bottom-up, attention-capturing nature of positive items, which may be attenuated when more strategic control is afforded to participants during encoding, especially in the self-regulated task of the current study where participants would be expected to require less time to implement strategies when they have full control over the presentation and study of items.

\section{Method}

Participants A new group of 54 younger adults (43 females, $M_{\text {age }}=20.44$ years, $S D_{\text {age }}=1.51$ years, age range: $18-27$ years) volunteered to participate in Experiment 2. Three participants were excluded from further analyses due to procedural error and missing data $(n=2)$ and outlying age $(n=1$, age: 42 years). As previously in Experiment 1, all younger adults were UCLA undergraduate students who participated for course credit. All younger adult participants presented with normal or corrected-to-normal vision, no physical disability, and clinically normal cognitive function. None of the participants in Experiment 1 participated in Experiment 2.

Materials and apparatus The items used as stimuli in this study were the same as those used previously in Experiment 1 (i.e., ten red and green, negatively and positively valued number-items, respectively). As in the previous experiment, the ten number-items, ranging from -25 to +25 in increments of 5 with no 0 value, were randomly placed within a $5 \times 5$ grid to form the eight unique grids used as the stimuli in this experiment.

Procedure Whereas participants in Experiment 1 were shown study grids with number-items presented either sequentially or simultaneously for $30 \mathrm{~s}$, participants in Experiment 2 were shown a blank grid for $30 \mathrm{~s}$ that was supported by interactive buttons representing each of the ten to-be-studied numberitems ranging from -25 to +25 (Fig. 5). Participants were 
instructed to choose the items to study for as long as they wanted, by pressing the buttons that displayed the corresponding number item and its associated location on the grid, for as long as the participant chose to view it. Participants were thus able to control which number-items they studied, as well as for how long to study each item (i.e., self-regulated learning), and were allowed to view each number item as many times as they desired during the study phase. These interactive number-item value buttons were vertically displayed on the screen directly to the left of the grid, with the presentation of the first, topmost values being counter-balanced across alternating grids. That is, participants would either begin with $+25,+20,+15, \ldots$ or $-25,-20,-15, \ldots$ as the presented top-most values for the button display, which were then flipped accordingly to the opposing format in an alternating fashion for each of the eight study grids.

Apart from the participant having full control over which randomly placed stimuli within the grid were studied, as well as the length of time they studied that item for, the procedures for Experiment 2 were identical to those of Experiment 1: participants were told to maximize their overall score (out of 75 points), and were shown a brief visual mask for $0.5 \mathrm{~s}$ before being asked to replace the items in their previously studied locations during the 30 -s self-regulated study period. Participants were given the same immediate feedback on their

\section{9 seconds remaining}

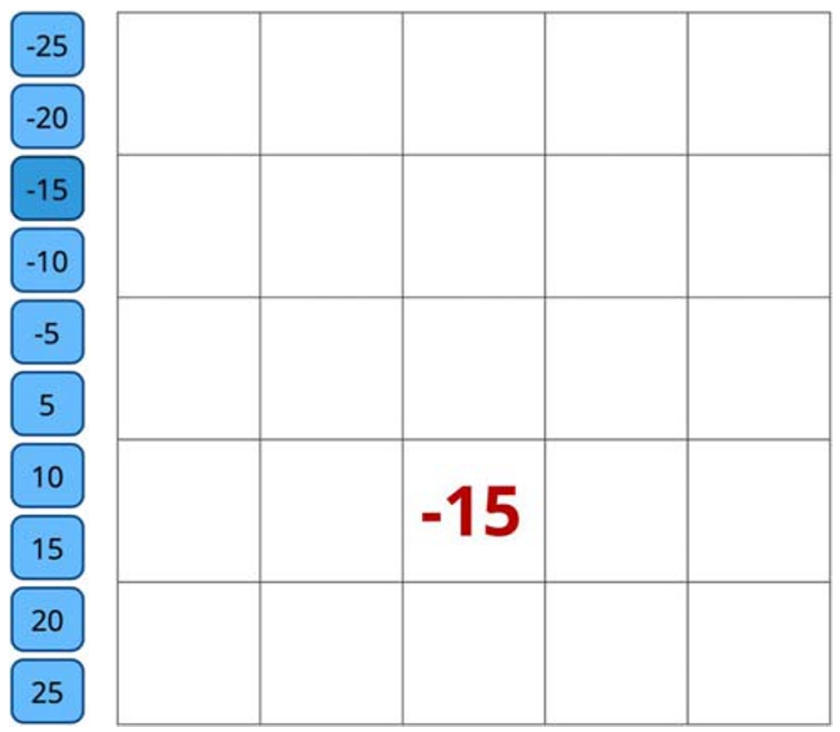

Fig. 5 An example of a grid that participants may have been presented with during the self-regulated study phase (Experiment 2 ). Ten values (five positive, five negative), ranging from -25 to +25 in increments of 5 , excluding 0 , were randomly assigned a location in the grid. No row or column has more than two values. Participants interacted with the buttons to the left of the grid to control which number-items they studied, as well as for how long each value was studied for with one item present in the grid at any point performance as in Experiment 1, after completing each studytest grid and repeated the process for all eight study-test cycles.

\section{Results}

Scoring performance As in Experiment 1, participants had the opportunity to score a minimum of -75 points and a maximum of +75 points in each study-test cycle. To examine their overall scoring performance with regard to grid number, we conducted a 1 (Presentation format: self-regulated) $\times 8$ (Grid number: $1,2, \ldots, 8)$ repeated-measures ANOVA on the overall points-scores (Fig. 6). This analysis revealed a significant main effect of grid number, $F(7,371)=2.58, p=.01, \eta^{2}=$ .05 . Post hoc paired-samples $t$-tests with a Bonferroni correction indicated that scores were higher on Grid $8(M=-1.10$, $S D=3.07)$ relative to Grid $1(M=-2.96, S D=3.15), t(53)=$ $3.43, p=.03$, and Grid $2(M=-2.94, S D=2.91), t(53)=3.38$, $p=.04$. No other comparisons were significant, adjusted $p \mathrm{~s}>$ .48. This suggests that participants scored more points at the end of the task relative to the beginning.

Similar to Experiment 1, we conducted the same polynomial regression predicting points-scores from grid number to follow-up on the significant main effect. The model was a significant predictor of points-scores, $R^{2}=.02, F(2,429)=$ $5.32, p=.01$. Both the coefficients for the intercept, $\beta_{0}=-$ $2.21, p<.001$, and the linear term, $\beta_{1}=.21, p=.001$, were significant predictors, while the quadratic term coefficient was not, $\beta_{2}=-.0004, p=.99$. Consistent with Experiment 1, this finding indicates a positive linear relationship between grid

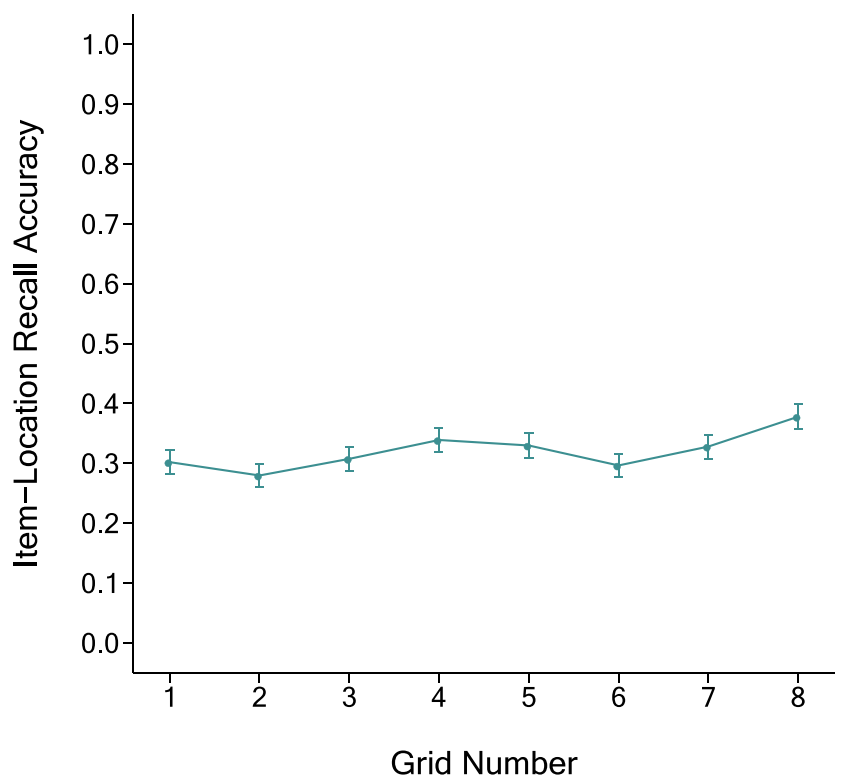

Fig. 6 The proportion of number-items correctly placed as a function of grid number when presented in the self-regulated study format in Experiment 2. Error bars represent \pm 1 standard error 
number and points such that with each increase in grid number the amount of points earned also increased.

Memory performance Memory performance for Experiment 2 was assessed in the same way that it was for Experiment 1 by investigating the ability of participants to correctly replace items into the exact target locations in which they were viewed in the prior study phase for each grid. This was first examined across grids and then as a function of item value.

Recall across grids To examine overall memory performance with increasing task-experience in the self-regulated presentation format, we conducted a 1 (Presentation format: self-regulated) $\times 8$ (Grid number: 1, 2, .., 8) repeated-measures ANOVA on the proportion of items correctly placed. This analysis revealed no main effect of grid number, $F(7,371)=$ $1.82, p=.08, \eta^{2}=.03$, suggesting that a consistent amount of information was recalled across trials regardless of item value.

Recall by item value To examine overall memory performance for each associated item value of the individual number-items in the self-regulated presentation format, we conducted a 1 (Presentation format: self-regulated) $\times$ 10 (Item value: $-25,-20, \ldots,+25$ ) repeated-measures ANOVA on the proportion of items correctly placed (Fig. 7). Mauchly's Test of Sphericity indicated that the assumption of sphericity had been violated, $\chi^{2}(44)=248.19, p<$ .001 ; therefore, a Greenhouse-Geisser $(\varepsilon=.419)$ correction was used. There was a significant main effect of item value, $F(3.77,199.76)=8.12, p<.001, \eta^{2}=.13$.

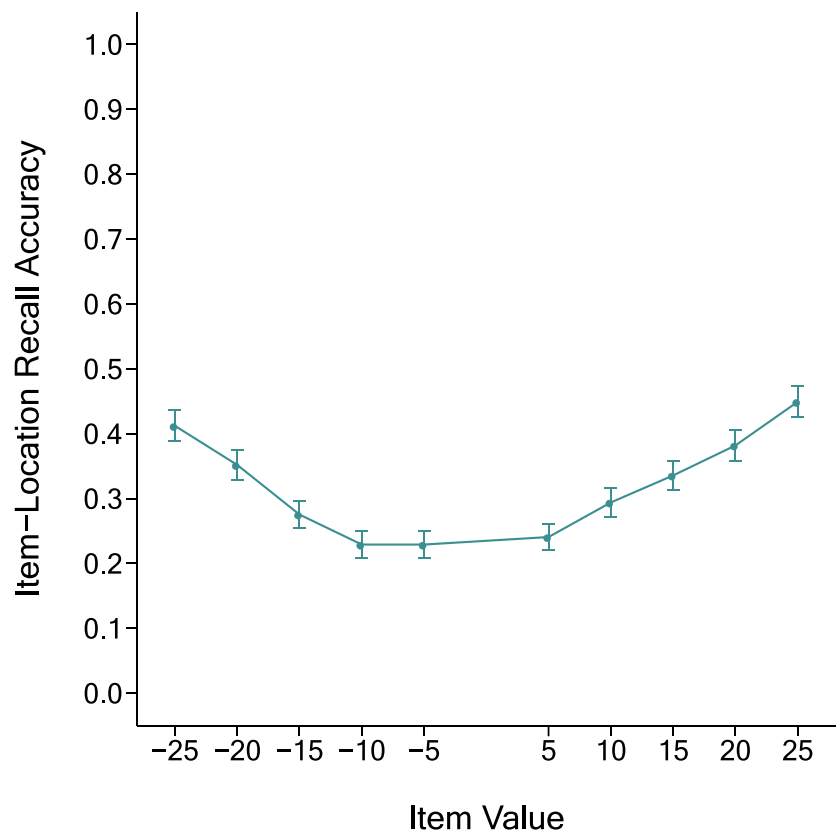

Fig. 7 The proportion of number-items correctly placed as a function of item value when presented in the self-regulated study format in Experiment 2. Error bars represent \pm 1 standard error
Similar to Experiment 1, we conducted linear and quadratic model fits for memory performance as a function of item value using a regression framework to avoid many post hoc comparisons. Tested linear models were of the following form: Recall Accuracy $=\beta_{0}+\beta_{1}$ (item value). Tested quadratic models were of the following form: Recall Accuracy $=\beta_{0}+$ $\beta_{1}$ (item value $)+\beta_{2}$ (item value $)^{2}$. The quadratic model was significant, $R^{2}=.97, F(2,9)=112.90, p<.001$, with both significant linear, $\beta_{1}=.25, p=.01$, and quadratic, $\beta_{2}=.95, p$ $<.001$, standardized coefficients indicating a U-shaped relationship between item value and recall accuracy.

Recall by sign We followed a similar procedure to that of Experiment 1 for examining the differences between positive and negative values of the same magnitude for each of our dependent measures. To determine whether there was a bias for positive or negative values, we conducted paired-samples $t$-tests with a Bonferroni correction. In contrast to Experiment 1 , there was no difference in recall accuracy between positive and negative items for any of the magnitudes, adjusted $p s>$ .30. Further, there was no difference in overall recall performance for positive $(M=.34, S D=.15)$ and negative $(M=.30$, $S D=.14)$ items, $t(53)=1.26, p=.21$.

Response order by item value As in Experiment 1, participants were allowed to replace items in the test grid in any order. To examine the order in which they replaced each item into the test grid, we conducted a 1 (Presentation format: selfregulated) $\times 10$ (Item value: $-25,-20, \ldots,+25)$ repeatedmeasures ANOVA on response order (Fig. 8). Mauchly's

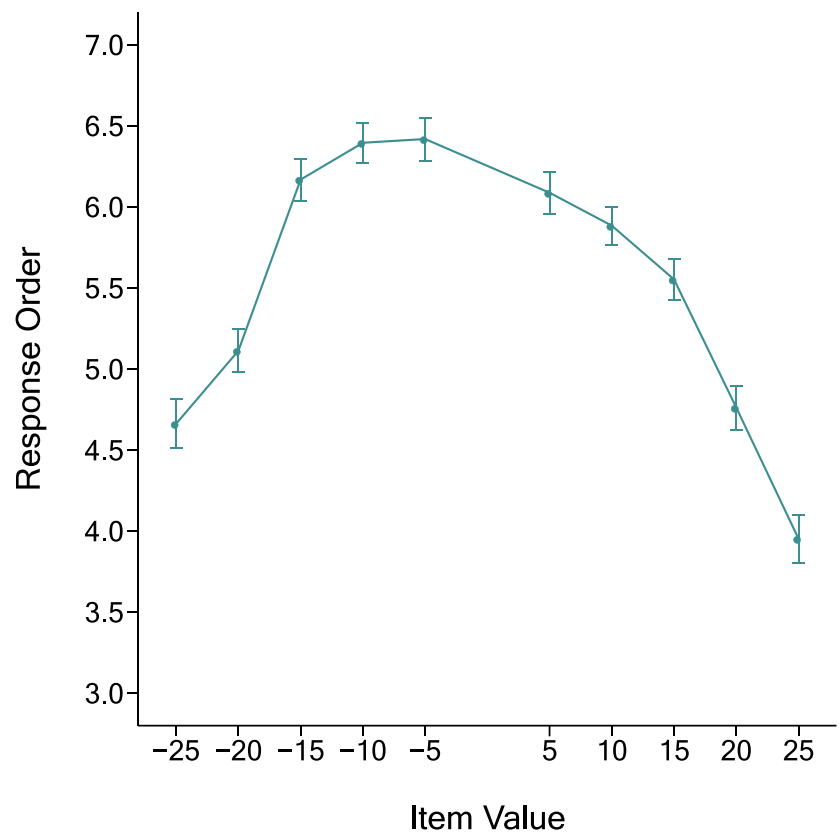

Fig. 8 Mean response order for values during the testing phase as a function of item value when presented in the self-regulated study format in Experiment 2. All error bars represent \pm 1 standard error 
Test of Sphericity indicated that the assumption of sphericity had been violated, $\chi^{2}(44)=335.79, p<.001$; therefore, a Greenhouse-Geisser $(\varepsilon=.357)$ correction was used. There was a significant main effect of item value, $F(3.22,170.40)$ $=17.01, p<.001, \eta^{2}=.24$.

To examine the overall trend, linear and quadratic models were fitted to the data. The quadratic model was significant, $R^{2}=.97, F(2,9)=99.60, p<.001$, with significant linear, $\beta_{1}$ $=-.31, p=.003$, and quadratic, $\beta_{2}=-.93, p<.001$, standardized coefficients indicating an inverted U-shaped relationship between item value and response order. As such, higher magnitude positive and negative items were both replaced earlier on in the testing phase relative to lower magnitude items. With regard to the particular sign, Bonferroni-adjusted paired-samples $t$-tests between items of the same magnitude indicated no significant differences in individual item values, adjusted $p s>.19$. Overall, however, there was a significant difference in the response order of positive $(M=5.25, S D=0.91)$ relative to negative $(M=5.75$, $S D=0.91)$ items, $t(53)=2.03, p=.047$, suggesting that positive items were replaced earlier than negative items.

Study frequency With the implementation of the selfregulated study paradigm, we were also able to examine the number of times participants studied each item as a function of its value. Study frequency was measured by averaging the number of study visits per item, per grid throughout the selfregulated study task. This was possible to do since the selfregulated study task gave participants control over (a) which items they studied, (b) the order in which they were studied, (c) for how long to study each item, and (d) how many times to study (or not study) each item. We conducted a 1 (Presentation format: self-regulated) $\times 8$ (Grid number: 1, 2, $\ldots, 8)$ repeated-measures ANOVA on study frequency and found no main effect of grid number, $F(7,371)=0.69, p=$ $.68, \eta^{2}=.013$, indicating that participants visited (studied) relatively the same number of items during each of the eight self-regulated study phase grids.

To determine the effects of item value on study frequency, we conducted a 1 (Presentation format: self-regulated) $\times 10$ (Item value: $-25,-20, \ldots,+25$ ) repeated-measures ANOVA on study frequency (Fig. 9). Mauchly's Test of Sphericity indicated that the assumption of sphericity had been violated, $\chi^{2}(44)=841.24, p<.001$; therefore, a Greenhouse-Geisser $(\varepsilon=.276)$ correction was used. We found a significant main effect of item value, $F(2.49,131.71)=21.80, p<.001, \eta^{2}=$ .29. Linear and quadratic models were fitted to examine overall trends. The quadratic model was significant, $R^{2}=.92, F(2$, $9)=40.25, p<.001$, with both significant linear, $\beta_{1}=.37, p=$ .01 , and quadratic, $\beta_{2}=.88, p<.001$, standardized coefficients indicating a U-shaped relationship between item value and study frequency. This suggests that participants studied higher magnitude items more frequently than lower magnitude items.
In terms of which sign was favored, Bonferroni-adjusted paired-samples $t$-tests indicated that the +25 and +20 items were studied more frequently than the -25 and -20 items, $t(53)=3.26, p=.01$, and $t(53)=2.83, p=.04$, respectively. There was no difference in study frequency between the other magnitudes, adjusted $p \mathrm{~s}>.05$. Overall, on average, positive items were studied more frequently $(M=2.12, S D=0.81)$ than negative items $(M=1.73, S D=0.71), t(53)=2.85, p=$ .01 .

Study time We were also able to examine the average length of time spent on each item when it was selected. Study time was measured by averaging the number of seconds that each item was viewed for, per study visit. We conducted a 1 (Presentation format: self-regulated) $\times 8$ (Grid number: 1, 2, $\ldots, 8)$ repeated-measures ANOVA on study frequency. Mauchly's Test of Sphericity indicated that the assumption of sphericity had been violated, $\chi^{2}(44)=54.81, p<.001$; therefore, a Greenhouse-Geisser $(\varepsilon=.790)$ correction was used. We found a significant main effect of grid number, $F(5.53,293.25)=3.29, p=.01, \eta^{2}=.06$. Follow-up pairedsamples $t$-tests with a Bonferroni correction indicated that participants' spent less time on each study visit on Grid 7 ( $M$ $=1.16, S D=0.43)$ relative to Grid $1(M=1.41, S D=0.56)$, $t(53)=3.65, p=.02$, and Grid $2(M=1.40, S D=0.52), t(53)=$ $3.86, p=.01$. No other comparisons were significant, adjusted $p \mathrm{~s}>.11$.

We conducted a 1 (Presentation format: self-regulated) $\times$ 10 (Item value: $-25,-20, \ldots,+25$ ) repeated-measures ANOVA on study time (Fig. 10). Mauchly's Test of Sphericity

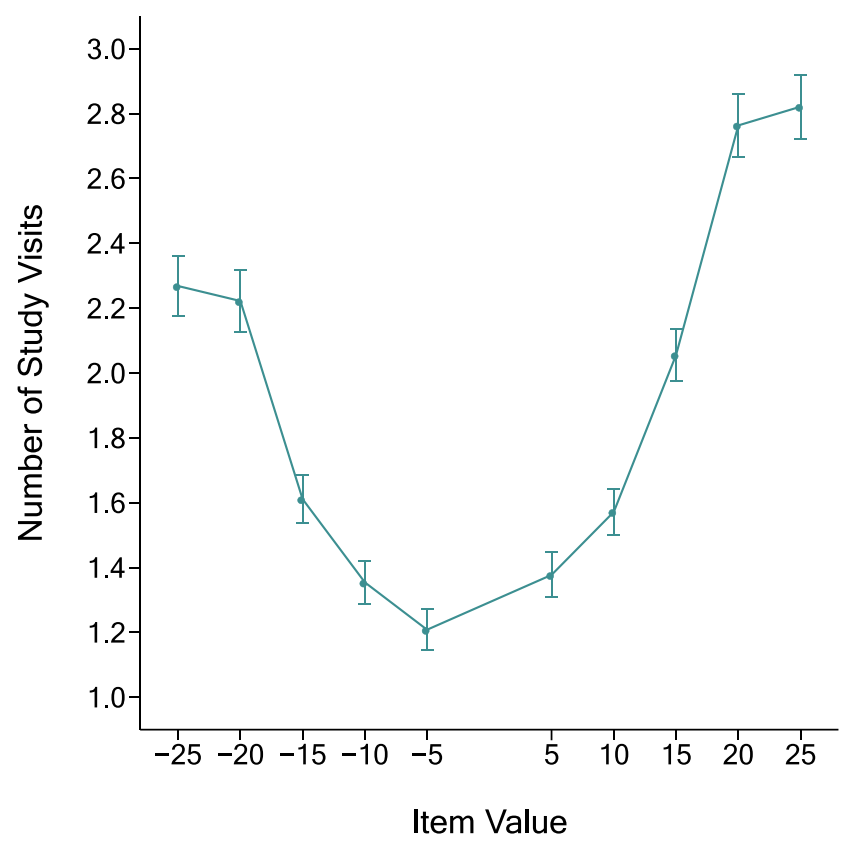

Fig. 9 Mean number of study visits (frequency) as a function of item value for self-regulated value-location associations in Experiment 2. All error bars represent \pm 1 standard error 
indicated that the assumption of sphericity had been violated, $\chi^{2}(44)=345.69, p<.001$; therefore, a Greenhouse-Geisser $(\varepsilon$ $=.498)$ correction was used. There was no main effect of item value, $F(4.48,237.31)=2.19, p=.06, \eta^{2}=.04$, suggesting that study time did not differ as a function of item value. When comparing between positive and negative items of the same magnitude, no significant differences were found, as indicated by the Bonferroni-adjusted paired-samples $t$-tests, adjusted $p \mathrm{~s}$ $>$.67. Overall, on average, positive $(M=1.34, S D=0.42)$ and negative $(M=1.26, S D=0.51)$ items were studied for the same amount of time per study visit, $t(53)=1.13, p=.26$.

\section{Discussion}

For information studied in the self-regulated presentation format, we found consistently higher recall accuracy for the highend positive and negative items compared to the low-end positive and negative items. The positivity preference exhibited in Experiment 1 was not present in participants' memory performance in this experiment despite participants replacing positive items earlier on during testing. However, there was a positivity preference when examining participants' study choices. While higher magnitude items were studied more frequently than lower magnitude items in general, there was an additional increase in study frequency for the most positive (i.e., the +20 and +25 ) items relative to the most negative (i.e., -20 and -25$)$ items. This positivity preference in study frequency, however, did not manifest in more study time per individual study visit for positive items. Contrary to the

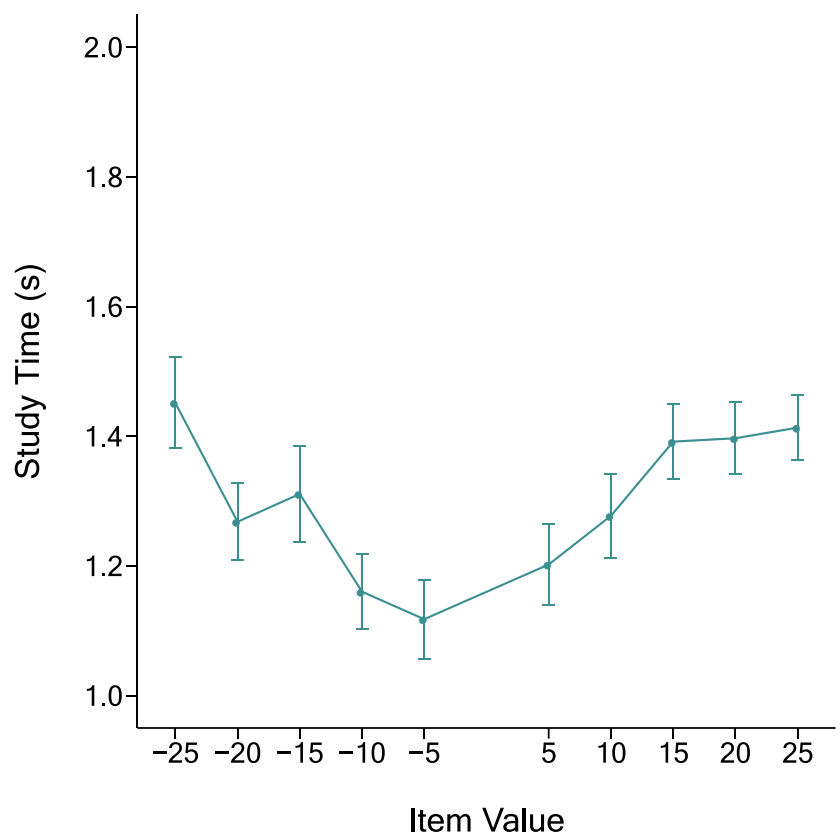

Fig. 10 Mean number of seconds spent studying values per visit during the testing phase as a function of item value when presented in the selfregulated study format in Experiment 2. All error bars represent \pm 1 standard error differences in recall for positive over negative items found in Experiment 1, these null differences in recall for positive and negative items in Experiment 2 may be due to an implicit averaging of the points-gained and loss-avoidance approaches, especially when more cognitive resources are available during encoding when participants are given more control over the presentation format and study strategy implementation. Overall, the results of Experiment 2 suggest that participants actively study positive information over negative information in the current study.

\section{General discussion}

Prior research investigating the role of value-directed remembering in the visuospatial domain established that items with a higher associated value were consistently better remembered compared to items with a lower value (Ariel et al., 2015; Castel et al., 2002; Middlebrooks et al., 2017; Siegel \& Castel, 2018a, 2018b). However, the opportunity to lose points in this kind of paradigm has not been well researched and may have critical implications for participants' study strategies when information varies in importance. Under various presentation formats in Experiment 1, participants adopted a selective strategy by remembering higher magnitude items over lower magnitude items. Yet, there was a positive memory preference in both presentation formats despite negative items having an equal influence on total scores. This focus on gaining points (as compared to avoiding losing points) was further explored in Experiment 2, where we were able to directly measure participants' study-time allocation given the employed experimental paradigm. In essence, the simultaneous presentation format of Experiment 1 and the interactive presentation format of Experiment 2 are both self-regulated study formats; however, participants in the simultaneous presentation format of Experiment 1 are presented with all stimuli at once within the visuospatial domain while participants in Experiment 2 only saw one item at a time (i.e., more similar to the sequential presentation format of Experiment 1). As such, the amount of perceived visual information that occurred all at once was different during the study phases of Experiments 1 and 2, which could have had downstream influences on the strategies that participants used to allocate study time to items of varying value. When more control was afforded to participants during study, their recall suggested a more measured approach, with equivalent memory for positive and negative items. This occurred even though participants studied positive items more frequently and output them earlier during the testing phase. Perhaps one explanation for the positivity bias during the self-regulated study phase of Experiment 2 not improving memory performance is the labor-in-vain effect, which suggests that increasing self-paced study time does not subsequently result in higher memory performance 
(Nelson \& Leonesio, 1988). Though participants in Experiment 2 were not found to have studied positive items longer than corresponding negative items, highly positive information was studied more frequently, for the same amount of time per study visit, compared to the other to-be-learned positive and negative information. An alternative explanation may be that the generally more arousing nature of negative information may have automatically captured participants' attention, compared to the less salient positive information, as found in an abundance of prior work (Bowen, Kark, \& Kensinger, 2018; Carretié, Hinojosa, Martín-Loeches, Mercado, \& Tapia, 2004; Clewett \& Murty, 2019; Eastwood, Smilek, \& Merikle, 2003; Hochman \& Yechiam, 2011; Kensinger \& Corkin, 2003a, 2003b; Mickley \& Kensinger, 2008; Mickley Steinmetz \& Kensinger, 2009; Siegel, Graup, \& Castel, 2020). Participants may have therefore been required to utilize their top-down strategic control by allocating more frequent study visits to positive items to match the salience of the corresponding negative items, producing equivalent memory for items of these two valences.

Additionally, this observed positivity preference may be driven by the regulatory fit theory (Higgins, 2005, 2006; Spiegel et al., 2004), as the phrasing of the prescribed task goals for participants to maximize their overall score may have influenced their experience of value within the current study. The results we observe seem to be more supported by the regulatory fit theory than by the prospect theory when attentional resources are more strained during encoding, in that the type of goal that participants pursued was primarily a points-gained approach (i.e., a promotion-focused goal orientation; Cesario et al., 2007; Higgins, 2006). In sum, when presented with information varying in positive and negative value, participants in this task adopted a points-gained approach, focusing on studying and remembering positive information over equally valued negative information.

Given that participants could selectively attend to and remember higher magnitudes relative to lower magnitudes, this finding replicates previous work suggesting participants are effective in prioritizing important associative information, even under attentionally demanding conditions (Ariel et al., 2015; Castel et al., 2002; Siegel \& Castel, 2018b). Such selectivity is emphasized by participants' study decisions in Experiment 2, with higher magnitudes receiving more study visits (and thus more total study time) despite equivalent study time per visit with lower magnitudes. As such, these results add further evidence that participants are effective in prioritizing important information, consistent with a large body of work demonstrating preserved selectivity with various materials like unrelated word pairs (Ariel et al., 2015), name-face pairs (Hargis \& Castel, 2017), medication side effects (Hargis \& Castel, 2018), and item-location pairs (Siegel \& Castel, 2018b), and under varying degrees of cognitive ability, like healthy aging (Castel et al., 2002; Hayes et al., 2013; Siegel \&
Castel, 2018a), Alzheimer's disease (Castel et al., 2009), attention-deficit hyperactivity disorder (Castel et al., 2011), and different working memory capacities (Griffin, Benjamin, Sahakyan, \& Stanley, 2019; Middlebrooks et al., 2017; Miller, Gross, \& Unsworth, 2019; Robison \& Unsworth, 2017).

Despite this ability to prioritize high-magnitude over lowmagnitude information, participants' strategy use was indeed flawed, as evidenced by the explicit focus on high-magnitude positive information. Prior to experimentation, we expected that participants would remember positive and negative information equally, consistent with prior work demonstrating that both rewards and punishments may produce equivalent memory enhancement (Castel et al., 2016; Madan \& Spetch, 2012; Madan et al., 2014; Shigemune et al., 2013). This would represent the optimal strategy in the task, as both positive and negative items equally contributed to participants' total score. In contrast, participants in the current study adopted a lessthan-optimal strategy by favoring positive items.

The findings of the current study also appear to be contradictory to prior work using sequential encoding conditions with rewards and punishments that did not find a positivity bias (Castel et al., 2016; Shigemune et al., 2013). One potential reason for the discrepancy between results may be the difficulty of the task in the current study. Although words were required to be bound to specific locations in Shigemune et al. (2013) and dollar values to faces in Castel et al. (2016), encoding difficulty in the current visuospatial binding task may have been greater for three potential reasons. First, the amount of potential locations in the current task $(25$ in the $5 \times 5)$ grid provided for a larger set of responses and lower chance performance than Shigemune et al. (2013) in which location options were either left or right. Secondly, the confusability of items in the current study was quite high, as items only differed in terms of their color (green for positive items and red for negative items) and magnitude ( -25 to +25 in increments of five). As such, discriminating between individual items may have been more difficult due to their perceptual and conceptual similarity, and subsequent item-location associative memory more challenging (e.g., "was it +25 in cell $(1,4)$ or +20 in cell $(1,5)$ ?") than when items were distinct, unrelated words paired to a left or right spatial location (e.g., "was it BANK-left or BANK-right?") in Shigemune et al. (2013) or when items were distinct faces paired with dollar values (e.g., "was it the woman with long hair who owed me $\$ 100$ or the bald man?") in Castel et al. (2016). Thirdly, the current study used a free-recall paradigm in which item-location associations needed to be retrieved entirely by the participant, in contrast to Shigemune et al. (2013), who used a recognition test and Castel et al. (2016), who used cued-recall testing. Free recall may be a more demanding process than cued recall or recognition as more encoding specificity is required to produce accurate performance (Tulving \& Thompson, 1973; Watkins \& Gardiner, 1979). Due to these factors, the sequential format in the current study may have 
been relatively more difficult compared to those in prior work finding a lack of positivity bias.

The task in general was indeed difficult, perhaps resulting in poor total point score performance in both experiments $\left(M_{\text {Seq }}=-2.90, M_{\text {Sim }}=-0.62\right.$, and $M_{\text {Self }}=-2.21$ out of a possible 75 points). Crucially, participants in the sequential condition produced the worst performance on the task while also displaying the highest positivity preference, as evidenced by the order of their responses. Self-regulated studiers did remember an equivalent amount of positive and negative information, but their study decisions still reflected a bias towards positive items. It is also important to note that affording participants more direct control over their study choices did not, at least numerically, improve their performance on the task relative to a simultaneous presentation of information, consistent with the idea that learners may not always adopt ideal strategies when study is self-regulated (for a review, see Dunlosky \& Ariel, 2011).

It is evident then, that in the context of the current experimental paradigm, participants gave preferential treatment to positive over negative information. Although correctly remembering the locations of negatively valued items was equally as important for attaining a high overall score, participants may have chosen to directly focus on a "points-gained" approach, as opposed to an "avoiding points lost" approach. This finding contrasts with predictions from prospect theory (Kahneman \& Tversky, 1979; Tversky \& Kahneman, 1992), which posits that losses may be weighted larger than gains. In the context of the current study, this may have resulted in a loss-avoidance-oriented approach (i.e., more focus on negative items) to studying associative information of differing value. Instead, the results suggest that when the opportunity to both lose and gain rewards is present, positive information may be attended to and remembered at the expense of negative information of a similar magnitude despite the obvious fault in this strategy use. Importantly, this was still the case when attentional resources were strained during encoding, suggesting that this positivity preference is still present under more demanding conditions.

Future research could also extend upon the self-regulated presentation format to develop two versions of the task: (1) similar to the current version (i.e., full control over which items to study, the order to study them, and for how long they are studied) and (2) a new version that only allows participants to control for study time and which items to study. For example, participants would see one item populated in the grid (similar to the sequential presentation format of Experiment 1) and would be allowed to advance to the next item as soon as they were ready to do so. The next item would thus be random (i.e., not directly selected by the control of the participant as it would have been in the self-regulated study task of Experiment 2). By forcing the presentation order of items in a new self-regulated study task where participants can still control for length of time of study, we could better understand the underlying processes of the sequential presentation format when participants do not know which item to next expect during encoding.

It may also be useful to directly ask participants after conclusion of the study which strategies they used to study information and whether or not they explicitly focused on a subset of the information. This may provide more explicit evidence of participants' awareness of their study strategies and the effects it may have had on their memory performance. Future research could even examine this points-gained approach under more demanding conditions like a dual-task paradigm in which a secondary task is to be completed during encoding (e.g., Middlebrooks et al., 2017; Siegel \& Castel, 2018b). Under these conditions in which resources are further strained, we would expect an even more pronounced positivity preference, which would support and extend the results of the current study.

Finally, it would be useful to explore the extent to which these results apply to more naturalistic memory contexts. The items which were the to-be-remembered stimuli in the current study were individual numerical values that were used due to their simplistic nature. In everyday life, we have to remember the locations of more visually complex items that differ in importance (i.e., a high-value item like car keys or a lowvalue item like a pen). It would be interesting to compare competing predictions from prospect theory and regulatory fit theory using more naturalistic materials. For example, when required to remember the location of one's hypothetical car keys, the framing of the goal may influence memory strategies. Prospect theory would predict better memory for the following loss-avoidance-oriented framing: "If I forget where I put my car keys, I could get fired because I'll be late for work," than the positively framed equivalent: "If I remember where I put my car keys, I'll make it to work on time and I'll remain gainfully employed," while regulatory fit theory would predict the opposite pattern of results. The results from the current study suggest that the latter framing would result in superior memory performance, but the naturalistic and personal nature of the task may result in different findings as the stakes may be higher for losses than gains in this real-world example. The current study intended to explore and shed light on the underlying mechanism in value-based strategy use for negative and positive information in a pared-down paradigm (e.g., minimizing potential confounds like participants' current employment status). A fruitful avenue for future work would be to examine the extension of these findings to more naturalistic memory contexts.

\section{Conclusion}

We sought to examine how the locations of both positive and negative important values might be selectively remembered in 
the visuospatial domain when studied in a sequential, simultaneous, or self-regulated presentation format. Participants needed to change their encoding strategy, to alleviate memory deficits when more attentional resources were required, depending on the format in which these items were presented during encoding. Participants more accurately recalled the locations of these important values in the simultaneous relative to the sequential condition. Additionally, positively valued items were better remembered relative to the negatively valued items of the same magnitude, representing an inefficient strategy given the equivalent influence of positive and negative items on participants' total scores. This positivity bias found in the current study expands upon prior work elucidating the positivity effect that is generally seen across the lifespan with successful aging (for a review, see Mather \& Carstensen, 2005) in a novel domain, visuospatial memory. This adds to some relatively recent research showing that younger adults, despite generally prioritizing negative information in cognitive processing (Baumeister, Bratslavsky, Finkenauer, \& Vohs, 2001; Rozin \& Royzman, 2001), can have positivity effects in memory when their goal orientation is shifted to focus on the present (Reed \& Carstensen, 2012; Sedikides \& Skowronski, 2020). When participants were given full control over which items they studied, the order in which they were studied, and the length of study time per item, their memory performance was equivalent between positive and negative items, but positive items were still studied more frequently and for more total time. Overall, while the current study finds further support for the value-directed remembering paradigm within the visuospatial domain when there is the potential to lose points, it also provides evidence for an inherent bias towards positively valued items despite the relatively equal role that the positive and negative items played in terms of task goals.

Acknowledgements This research was supported in part by the National Institutes of Health (National Institute on Aging; Award Number R01 AG044335 to Alan Castel). The authors would like to thank Mary Hargis, Mary Whatley, and the rest of the members of the Memory and Lifespan Cognition Laboratory at UCLA, as well as Naomi Eisenberger and Terri and Bruce Schwartz, for their guidance and support throughout this project.

\section{References}

Ariel, R., \& Dunlosky, J. (2013). When do learners shift from habitual to agenda-based processes when selecting items for study? Memory \& Cognition, 41, 416-428.

Ariel, R., Dunlosky, J., \& Bailey, H. (2009). Agenda-based regulation of study-time allocation: when agendas override item-based monitoring. Journal of Experimental Psychology: General, 138, 432.
Ariel, R., Price, J., \& Hertzog, C. (2015). Age-related associated memory deficits in value-based remembering: The contribution of agendabased regulated and strategy use. Psychology and Aging, 30, 795808.

Baumeister, R. F., Bratslavsky, E., Finkenauer, C., \& Vohs, K. D. (2001). Bad is stronger than good. Review of General Psychology, 5, 323370.

Bowen, H. J., Kark, S. M., \& Kensinger, E. A. (2018). NEVER forget: negative emotional valence enhances recapitulation. Psychonomic Bulletin \& Review, 25, 870-891.

Carretié, L., Hinojosa, J. A., Martín-Loeches, M., Mercado, F., \& Tapia, M. (2004). Automatic attention to emotional stimuli: neural correlates. Human Brain Mapping, 22, 290-299.

Castel, A. D. (2008). The adaptive and strategic use of memory by older adults: Evaluative processing and value-directed remembering. In A. S. Benjamin \& B. H. Ross (Eds.), The psychology of learning and motivation (Vol. 48, pp. 225-270). London, UK: Academic Press.

Castel, A. D., Balota, D. A., \& McCabe, D. P. (2009). Memory efficiency and the strategic control of attention at encoding: Impairments of value-directed remembering in Alzheimer's disease. Neuropsychology, 23, 297.

Castel, A. D., Benjamin, A. S., Craik, F. I. M., \& Watkins, M. J. (2002). The effects of aging on selectivity and control in short-term recall. Memory \& Cognition, 30, 1078-1085.

Castel, A. D., Friedman, M. F., McGillivray, S., Flores, C. C., Murayama, K., Kerr, T., \& Drolet, A. (2016). I owe you: Age-related similarities and differences in associative memory for gains and losses. Aging, Neuropsychology, and Cognition, 23, 549-565.

Castel, A. D., Lee, S. S., Humphreys, K. L., \& Moore, A. N. (2011). Memory capacity, selective control, and value-directed remembering in children with and without attention-deficit/hyperactivity disorder (ADHD). Neuropsychology, 25, 15-24.

Castel, A. D., Murayama, K., Friedman, M. C., McGillivray, S., \& Link, I. (2013). Selecting valuable information to remember: Age-related differences and similarities in self-regulated learning. Psychology and Aging, 28, 232-242.

Cesario, J., Higgins, E. T., \& Scholer, A. A. (2007). Regulatory fit and persuasion: Basic principles and remaining questions. Social and Personality Psychology Compass, 2, 444-463.

Chalfonte, B. L., \& Johnson, M. K. (1996). Feature memory and binding in young and older adults. Memory \& Cognition, 24, 403-416.

Clewett, D., \& Murty, V. P. (2019). Echoes of emotions past: How neuromodulators determine what we recollect. Eneuro, 6(2).

Cohen, M. S., Rissman, J., Suthana, N. A., Castel, A. D., \& Knowlton, B. J. (2014). Value-based modulation of memory encoding involves strategic engagement of fronto-temporal semantic processing regions. Cognitive, Affective, \& Behavioral Neuroscience, 14, 578592.

Cooper, J. C., \& Knutson, B. (2008). Valence and salience contribute to nucleus accumbens activation. Neuroimage, 39, 538-547.

Dunlosky, J., \& Ariel, R. (2011). Self-regulated learning and the allocation of study time. In B. H. Ross (Ed.), Psychology of learning and motivation (Vol. 54, pp. 103-140). San Diego, CA: Elsevier.

Dunlosky, J., Ariel, R., \& Thiede, K. W. (2011). Agenda-based regulation of study-time allocation. In P. Higham (Ed.), Constructions of Remembering and Metacognition (pp. 182-198). Hampshire, England: Palgrave Macmillan.

Dunlosky, J., \& Thiede, K. W. (2004). Causes and constraints of the shiftto-easier-materials effect in the control of study. Memory \& Cognition, 32, 779-788.

Eastwood, J. D., Smilek, D., \& Merikle, P. M. (2003). Negative facial expression captures attention and disrupts performance. Perception \& Psychophysics, 65, 352-358.

Elliott, B. L., \& Brewer, G. A. (2019). Divided attention selectively impairs value-directed encoding. Collabra: Psychology, 5, 4. 
Gallo, D. A., Sullivan, A. L., Daffner, K. R., Schacter, D. L., \& Budson, A. E. (2004). Associative recognition in Alzheimer's disease: evidence for impaired recall-to-reject. Neuropsychology, 18, 556-563.

Griffin, M. L., Benjamin, A. S., Sahakyan, L., \& Stanley, S. E. (2019). A matter of priorities: High working memory enables (slightly) superior value-directed remembering. Journal of Memory and Language, 108, 104032.

Hargis, M. B., \& Castel, A. D. (2017). Younger and older adults' associative memory for social information: The role of information importance. Psychology and Aging, 32, 325-330.

Hargis, M. B., \& Castel, A. D. (2018). Younger and older adults' associative memory for medication interactions of varying severity. Memory, 26, 1151-1158.

Hayes, M. G., Kelly, A. J., \& Smith, A. D. (2013). Working memory and the strategic control of attention in Older and Younger Adults. The Journals of Gerontology, Series B: Psychological Sciences and Social Sciences, 68, 176-183.

Higgins, E. T. (2005). Value from regulatory fit. Current Directions in Psychological Science, 14, 209-213.

Higgins, E. T. (2006). Value from hedonic experience and engagement. Psychological Review, 113, 439.

Hochman, G., \& Yechiam, E. (2011). Loss aversion in the eye and in the heart: The autonomic nervous system's responses to losses. Journal of Behavioral Decision Making, 24, 140-156.

Kahneman, D., \& Tversky, A. (1979). Prospect theory: An analysis of decision under risk. Econometrica, 47, 263-291.

Kane, M. J., Hambrick, D. Z., Tuholski, S. W., Wilhelm, O., Payne, T. W., \& Engle, R. W. (2004). The generality of working memory capacity: A latent-variable approach to verbal and visuospatial memory span and reasoning. Journal of Experimental Psychology: General, 133, 189-217.

Kensinger, E. A., \& Corkin, S. (2003a). Effect of negative emotional content on working memory and long-term memory. Emotion, 3, 378

Kensinger, E. A., \& Corkin, S. (2003b). Memory enhancement for emotional words: Are emotional words more vividly remembered than neutral words? Memory \& Cognition, 31, 1169-1180.

Madan, C. R., Ludvig, E. A., \& Spetch, M. L. (2014). Remembering the best and worst of times: Memories for extreme outcomes bias risky decisions. Psychonomic Bulletin \& Review, 21, 629-636.

Madan, C. R., \& Spetch, M. L. (2012). Is the enhancement of memory due to reward driven by value or salience? Acta Psychologica, 139, 343-349.

Mather, M., \& Carstensen, L. L. (2005). Aging and motivated cognition: The positivity effect in attention and memory. Trends in cognitive sciences, 9(10), 496-502.

Mickley, K. R., \& Kensinger, E. A. (2008). Emotional valence influences the neural correlates associated with remembering and knowing. Cognitive, Affective, \& Behavioral Neuroscience, 8, 143-152.

Mickley Steinmetz, K. R., \& Kensinger, E. A. (2009). The effects of valence and arousal on the neural activity leading to subsequent memory. Psychophysiology, 46, 1190-1199.

Middlebrooks, C. D., \& Castel, A. D. (2018). Self-regulated learning of important information under sequential and simultaneous encoding conditions. Journal of Experimental Psychology: Learning, Memory, and Cognition, 44, 779-792.

Middlebrooks, C. D., Kerr, T., \& Castel, A. D. (2017). Selectively distracted: Divided attention and memory for important information. Psychological Science, 28, 1103-1115.

Miller, A. L., Gross, M. P., \& Unsworth, N. (2019). Individual differences in working memory capacity and long-term memory: The influence of intensity of attention to items at encoding as measured by pupil dilation. Journal of Memory and Language, 104, 25-42.

Mitchell, S. H., \& Wilson, W. B. (2010). The subjective value of delayed and probabilistic outcomes: Outcome size matters for gains but not for losses. Behavioural Processes, 83, 36-40.

Naveh-Benjamin, M. (2000). Adult age differences in memory performance: tests of an associative deficit hypothesis. Journal of Experimental Psychology: Learning, Memory, and Cognition, 26, 1170-1187.

Nelson, T. O., \& Leonesio, R. J. (1988). Allocation of self-paced study time and the "labor-in-vain effect.". Journal of experimental psychology: Learning, Memory, and Cognition, 14, 676.

Nelson, T. O., \& Narens, L. (1990). Metamemory: A theoretical framework and new findings. In G. H. Bower (Ed.), The psychology of learning and motivation (Vol. 26, pp. 125-141). New York, NY: Academic Press.

Nguyen, L. T., Marini, F., Zacharczuk, L., Llano, D. A., \& Mudar, R. A. (2019). Theta and alpha band oscillations during value-directed strategic processing. Behavioural Brain Research, 367, 210-214.

Old, S. R., \& Naveh-Benjamin, M. (2008). Differential effects of age on item and associative measures of memory: a meta-analysis. Psychology and Aging, 23, 104-118.

Park, D. C., Lautenschlager, G., Hedden, T., Davidson, N. S., Smith, A. D., \& Smith, P. K. (2002). Models of visuospatial and verbal memory across the adult lifespan. Psychology and Aging, 17, 299-320.

Reed, A. E., \& Carstensen, L. L. (2012). The theory behind the agerelated positivity effect. Frontiers in Psychology, 3, 339.

Robison, M. K., \& Unsworth, N. (2017). Working memory capacity, strategic allocation of study time, and value-directed remembering. Journal of Memory and Language, 93, 231-244.

Rozin, P., \& Royzman, E. B. (2001). Negativity bias, negativity dominance, and contagion. Personality and Social Psychology Review, 5, 296-320.

Sedikides, C., \& Skowronski, J. J. (2020). In Human Memory, Good Can Be Stronger Than Bad. Current Directions in Psychological Science, 29, 86-91.

Shigemune, Y., Tsukiura, T., Kambara, T., \& Kawashima, R. (2013). Remembering with gains and losses: Effects of monetary reward and punishment on successful encoding activation of source memories. Cerebral Cortex, 24, 1319-1331.

Shing, Y. L., Werkle-Bergner, M., Brehmer, Y., Müller, V., Li, S., \& Lindenberger, U. (2010). Episodic memory across the lifespan: The contributions of strategic and associative components. Neuroscience and Biobehavioral Reviews, 34, 1080-1091.

Siegel, A. L. M., \& Castel, A. D. (2018a). The role of attention in remembering important item-location associations. Memory \& Cognition, 46, 1248-1262.

Siegel, A. L. M., \& Castel, A. D. (2018b). Memory for important itemlocation associations in younger and older adults. Psychology and Aging, 33, 30-45.

Siegel, A. L. M., Graup, R. S., \& Castel, A. D. (2020). Emotion-enhanced binding of numerical information in younger and older adults. Quarterly Journal of Experimental Psychology, 73, 134-145.

Spiegel, S., Grant-Pillow, H., \& Higgins, E. T. (2004). How regulatory fit enhances motivational strength during goal pursuit. European Journal of Social Psychology, 34, 39-54.

Stefanidi, A., Ellis, D. M., \& Brewer, G. A. (2018). Free recall dynamics in value-directed remembering. Journal of Memory and Language, 100, 18-31.

Thomas, A. K., Bonura, B. M., Taylor, H. A., \& Brunyé, T. T. (2012). Metacognitive monitoring in visuospatial working memory. Psychology and Aging, 27, 1099-1110. 
Tulving, E., \& Thompson, D. M. (1973). Encoding specificity and retrieval processes in episodic memory. Psychological Review, 80, 352-373.

Tversky, A., \& Kahneman, D. (1992). Advances in prospect theory: Cumulative representation of uncertainty. Journal of Risk and Uncertainty, 5, 297-323.

Watkins, M. J., \& Gardiner, J. M. (1979). An appreciation of generaterecognize theory of recall. Journal of Verbal Learning and Verbal Behavior, 18, 687-704.

Wong, S., Irish, M., Savage, G., Hodges, J. R., Piguet, O., \& Hornberger, M. (2019). Strategic value-directed learning and memory in Alzheimer's disease and behavioural-variant frontotemporal dementia. Journal of neuropsychology, 13(2), 328-353.
Zink, C. F., Pagnoni, G., Martin-Skurski, M. E., Chappelow, J. C., \& Berns, G. S. (2004). Human striatal responses to monetary reward depend on saliency. Neuron, 42, 509-517.

Open Practices Statement The data and materials for these experiments are available upon reasonable request. None of the experiments were preregistered.

Publisher's note Springer Nature remains neutral with regard to jurisdictional claims in published maps and institutional affiliations. 